noticed, and 3) in psychological standpoint, self-confidence and brighteness regain and mental test (WISC) revealed an increase in IQ from 77 to 102.

II. Irritable behavioral disorders in epilepsy:

Seven years old Japanese girl (H.S.) had complained of convulsion (left half of the body) and violent behavioral disorders under the medication of Ospolot (0.4), Aleviatin (0.15), Luminal (0.05), Tegretol (0.4), Wintamin (0.025) and others. Since Forel-H-tomy was performed at the right side over 11 months ago, remarkable improvements have been obtained under none of the medication.

1) Clinical seizures have disappeared and EEG abnormalities of the right hemisphere (siffuse dysrhythmia) have been also improved. 2) Preoperative character disorders such as aggressiveness, impulsiveness and unsteadiness disappeared. Mental test, which could be hardly performed before surgery because of her incorperation revealed an improvement of mental condition and also IQ after surgery.

\title{
7. Electrophysiological Observations during Stereotaxic Amygdalotomy
}

H. Narabayashi M. D., and R. Matsumoto M. D. and Chihiro OHYE

Eepartment of Neurology and Department of Neurosurgery, Juntendo University

Departmentof Neurosurgery, Gunma University

\section{The Effects of Septal Lesion on the Operant Behavior of the Rabbit}

\author{
Tetsuya Sakamoto, Ryungchan Kwak, Yoshinobu Okudarra, \\ Keiki Uneoka and Jiro SuzukI \\ Division of Neurosurgery, Institute of Brain Diseases, \\ Tohoku University School of Medicine, Sendai
}

Effects of bilateral septal lesions in the rabbits were examined with Skinner box in bar pressing for food on the schedule of the continuous reinforcement (CRF) and the differential reinforcement of the low rate $10 \mathrm{sec}$. (DRL). Thirty-six adult male rabbits of about $3000 \mathrm{gr}$ were used. Animals were trained for $30 \mathrm{~min}$. a day as a session.CRF learning was regarded as perfection when animals were reinforced over 100, and continuously DRL learning was performed up to getting stabilized reinforcement rate. The lesions were produced electrolytically through a bipolar stainless steel electrode which was positioned in the brain at A $4.0 \mathrm{~mm}, \mathrm{~L} 1.5 \mathrm{~mm}$ 